\title{
Exposição ao Mercúrio Orgânico em Populações Ribeirinhas do Alto Madeira, Rondônia, 1991: Resultados Preliminares ${ }^{1}$
}

\author{
Exposure to Organic Mercury in Riparian Populations on the Upper \\ Madeira River, Rondonia, Brazil, 1991: Preliminary Results
}

\begin{abstract}
Ana Amelia P. Boischio ${ }^{2}$
Antonio Barbosa ${ }^{3}$

BOISCHIO, A. A. P. \& BARBOSA, A. Exposure to Organic Mercury in Riparian Populations on the Upper Madeira River, Rondonia, Brazil, 1991: Preliminary Results. Cad. Saúde Públ., Rio de Janeiro, 9 (2): 155-160, Apr/Jun, 1993.
\end{abstract}

This study analyzes mercury concentration in hair samples obtained from the population living in the Upper Madeira River basin, Rondonia. Out of 311 hair samples analyzed, $51 \%(n=158)$ had a mercury concentration of over $10 \mathrm{ppm}$. The wide variability of mercury concentration is well-illustrated by a family in which there were 5 cases with the highest mercury concentrations (90.6 to $303.1 \mathrm{ppm}$ ), while in another 5 household members, the concentration ranged from 7.0 to 13.3 ppm. Special atention must be paid to women in the childbearing age, since the fetus is more vulnerable to lower mercury concentrations. $53 \%(n=37)$ females in the reproductive age bracket (age group 4) displayed a mercury concentration above $10 \mathrm{ppm}$.

Key words: Environmental Exposure; Mercury; Hair; Analysis; Fish Consumption

\section{INTRODUÇÃO}

O mercúrio metálico tem sido utilizado nas atividades garimpeiras na Amazônia graças à sua propriedade de formar amálgama, tornando possível a extração do ouro granulométrico disperso no sedimento de fundo, como ocorre no leito do Alto Madeira. Depois de utilizado, o mercúrio é lançado no ambiente em forma de vapor (durante a queima do amálgama) e em forma líquida (perdas no manuseio do carpete). O vapor de mercúrio é oxidado e retorna ao ambiente aquático, onde fica sujeito aos processos de organificação, cujo produto é, então,

\footnotetext{
${ }^{1}$ Pesquisa financiada pelo Conselho Nacional de Desenvolvimento Científico e Tecnológico, com apoio da Universidade Federal de Rondônia, do Instituto Brasileiro do Meio Ambiente e dos Recursos Naturais Renováveis (Ibama-RO) e Sedam (Governo do Estado de Rondônia).

${ }^{2}$ School of Public and Environmental Affairs, Indiana University, Bloomington, IN 47406, U.S.A.

${ }^{3}$ Departamento de Química da Universidade de Brasília. Campus Universitário, Brasília-DF, 70910-900, Brasil.
}

mobilizado nas cadeias alimentares aquáticas (Pfeiffer et al., 1990).

Fatores ecológicos tais como $\mathrm{pH}$, condutividade elétrica, disponibilidade de oxigênio, temperatura, atividade biológica e concentração de nutrientes, entre outros, são relevantes nos processos de organificação do mercúrio no ambiente (Fagerstrom et al., 1972).

As populações ribeirinhas da Amazônia que caracteristicamente utilizam o pescado local como principal item alimentar estão seriamente ameaçadas pelo mercúrio orgânico que se concentra nos diferentes níveis tróficos das cadeias alimentares. São inúmeras as variáveis que interferem na exposição destes indivíduos ao mercúrio, relacionadas tanto ao comportamento do mercúrio nesta cadeia trófica (por exemplo, biomagnificação) quanto ao consumo de pescado (espécies consumidas, sazonalidade e locais de captura).

No homem o sistema nervoso central é o principal órgão atingido pelo mercúrio orgânico. Sintomas clínicos em adultos incluem parestesia (alterações sensoriais), ataxia (falta de coorde- 
nação nos movimentos) e disartria (dificuldade na articulação das palavras), além de distúrbios visuais e auditivos. A ocorrência dos sintomas clínicos é dose-dependente (IPCS, 1990).

$\mathrm{O}$ mercúrio orgânico ultrapassa facilmente a barreira placentária, sendo que o feto é mais sensível a menores concentrações de mercúrio do que os adultos. O quadro clínico típico das crianças geradas sob tais exposições é também dose-dependente e inclui microcefalia, hiperreflexia e deficiência visual, auditiva, mental e motora (IPCS, 1990).

O presente trabalho tem como objetivo estabelecer os níveis de exposição ao mercúrio orgânico destas populações ribeirinhas. Para tanto, foram investigadas 150 famílias dentre as 1.100 existentes ao longo de $170 \mathrm{~km}$ do rio Madeira, a jusante de Porto Velho, capital do estado de Rondônia, incluindo a Reserva Ecológica do Cuniã. As amostras de cabelo aqui apresentadas, provenientes de 311 indivíduos, foram precedidas de entrevistas domiciliares, cujo objetivo principal consistiu em avaliar características do consumo de peixe, além de medições antropométricas (peso e altura). Considerando-se que o mercúrio é associado às proteínas do cabelo no momento em que o mesmo está sendo formado (WHO, 1976), estas amostras foram coletadas a partir do escalpo e apresentam comprimento entre 2,0 e 54,0 centímetros.

\section{PROCEDIMENTO ANALÍTICO}

Para as presentes análises, foi utilizado o método para determinação de mercúrio com vapor frio modificado por Magos et al. (1972), que utiliza a pré-digestão básica das amostras. Vários fios de cabelo, perfazendo uma massa de 10 a $15 \mathrm{mg}$, são picotados e colocados em um frasco reacional juntamente com hidróxido de sódio (45\%) e cisteína (1\%), procedendo-se ao aquecimento em chapa aquecedora, a $90^{\circ} \mathrm{C}$, durante 15 minutos. Após o resfriamento em recipiente contendo gelo moído, recompõe-se com água destilada a perda por volatilização, via pesagem, e o volume é completado para 10 $\mathrm{ml}$ com cloreto de sódio (1\%). Em seguida, são adicionados em um reator, a $1 \mathrm{ml}$ da amostra pré-digerida, cisteína (para complexar o mercú- rio e, assim, evitar perdas), cloreto de sódio ( $1 \%$, como eletrólito suporte), ácido sulfúrico $(16 \mathrm{~N})$ e hidróxido de sódio (45\%). Uma solução contendo cloreto estanoso e cloreto de cádmio é usada para reduzir os compostos de mercúrio a mercúrio elementar. A capacidade do cloreto de cádmio de quebrar as ligações carbono mercúrio possibilita a determinação do mercúrio total, incluindo a forma orgânica. Para se avaliar apenas o mercúrio presente sob a forma inorgânica, repete-se a mesma operação, mas usando-se como redutor apenas o cloreto estanoso. O mercúrio reduzido é vaporizado com ajuda da reação exotérmica ocorrida no reator e aspirado por meio de uma bomba, através do Monitor de Absorção Atômica (LDC Analytical, modelo 1255). As leituras de absorção são feitas por meio de um milivoltímetro digital. A concentração de mercúrio orgânico presente na amostra de cabelo é obtida pela diferença entre os teores de mercúrio total e mercúrio inorgânico determinados. Para obtenção dos resultados, é utilizado um padrão interno de mercúrio de $30 \mathrm{ng} / \mathrm{ml}$.

\section{RESULTADOS E DISCUSSÃO}

Dentre as 311 amostras de cabelo analisadas, 142 amostras - todas com concentração de mercúrio total superior a $10 \mathrm{ppm}$ - foram submetidas a análise para mercúrio inorgânico. Destas, $70 \%(\mathrm{n}=99)$ apresentam mais de $80 \%$ do mercúrio total na forma de mercúrio orgânico.

A população em estudo foi dividida segundo grupos etários, conforme mencionado na legenda da Tabela 1, que apresenta os intervalos de concentração de mercúrio total em cabelo de acordo com grupo etário. Observa-se que 51\% $(n=158)$ dos indivíduos revelam concentrações de mercúrio total em amostras de cabelo superiores a $10 \mathrm{ppm}$.

Do ponto de vista de saúde pública, especial atenção deve ser dirigida às mulheres em idade reprodutiva (grupo etário 4), onde 53\% (n=37) das mulheres investigadas apresentaram concentração de mercúrio superior a $10 \mathrm{ppm}$, sendo que em $17(24 \%)$ a concentração foi acima de $15 \mathrm{ppm}$. Na mesma perspectiva, crianças menores de 24 meses que ainda não consomem peixe 
TABELA 1. Intervalos de Concentração de Mercúrio em Amostras de Cabelo (ppm) dos Indivíduos Distribuídos por Grupo Etário na População Ribeirinha do Alto Madeira, Brasil, 1991

\begin{tabular}{|c|c|c|c|c|c|c|c|}
\hline \multirow{2}{*}{$\begin{array}{l}\text { Grupos } \\
\text { Etários }\end{array}$} & \multicolumn{6}{|c|}{ Intervalos de Concentração de Mercúrio } & \multirow{2}{*}{ Subtotal } \\
\hline & $<10$ & $10-15$ & $15-30$ & $30-50$ & $50-125$ & $>125$ & \\
\hline 1 & 19 & 4 & 1 & 0 & 0 & 0 & 24 \\
\hline 2 & 23 & 5 & 9 & 1 & 0 & 1 & 39 \\
\hline 3 & 45 & 21 & 18 & 5 & 1 & 2 & 92 \\
\hline 4 & 33 & 20 & 14 & 1 & 1 & 1 & 70 \\
\hline 5 & 12 & 9 & 16 & 6 & 2 & 0 & 45 \\
\hline 6 & 12 & 5 & 4 & 0 & 0 & 0 & 21 \\
\hline 7 & 9 & 2 & 6 & 3 & 0 & 0 & 20 \\
\hline Total & 153 & 66 & 68 & 16 & 4 & 4 & 311 \\
\hline
\end{tabular}

\section{Legenda para Grupo Etário:}

1 menores de 24 meses que não consomem peixe

2 bebês que já consomem peixe até 5 anos de idade

3 de 5 a 15 anos de idade

4 de 15 a 49 anos de idade, feminino

5 de 15 a 49 anos, masculino

6 maiores de 49 anos, feminino

7 maiores de 49 anos, masculino

(Obs.: Os intervalos são fechados nos limites inferiores)

(grupo etário 1), apresentaram concentrações de mercúrio acima de 10 ppm em 5 casos $(21 \%)$. Tais concentrações refletem as atuais exposições intra-uterinas e as exposições através do aleitamento materno.

Foram detectados oito casos (3\%) de exposições elevadas - com concentrações de mercúrio acima de $50 \mathrm{ppm}$ - , sendo que o máximo verificado foi de 303,1 ppm de mercúrio total (S.G.M., 13 anos, sexo feminino, $15,0 \mathrm{~cm}$ de comprimento de cabelo). Os cinco casos detectados acima de 90 ppm são da mesma família e referem-se a crianças de 4 a 13 anos de idade (com comprimentos de cabelo entre 8,0 e $15,0 \mathrm{~cm}$ ), além da própria mãe (M.J.R., 31 anos), com 145,0 ppm de mercúrio no cabelo $(43,0 \mathrm{~cm})$. Outros cinco indivíduos na mesma família apresentaram concentrações de mercúrio entre 7,0 ppm (M.G.M., 21 meses de idade, que já consome peixe, conforme informação referida, com 10,0 cm de cabelo) e 13,3 ppm (F.G.M., 35 anos, masculino, 4,5 cm). Outras idades com concentrações inferiores a 11,0 ppm variam entre 3 e 14 anos (5,5 a 9,0 cm de cabelo).

Estes dados ilustram a ampla variação nas exposições ao mercúrio, ainda que na mesma unidade familiar. Observa-se que todos os cinco casos de concentração de mercúrio acima de 90 ppm foram verificados em amostras de comprimento de cabelo igual ou superior a $8,0 \mathrm{~cm}$. No entanto, duas crianças desta mesma unidade familiar, com comprimentos de cabelo idênticos $(8,0 \mathrm{~cm})$ apresentaram 7,4 ppm (F.G.M., 4 anos, masculino) e 155,9 ppm (M.G.M., 3 anos, masculino) de mercúrio total. Importa saber quais as causas específicas de tais divergências. Por exemplo, foi observado, durante o trabalho 
de campo, que uma única refeição pode incluir até cinco espécies diferentes de peixe. A distribuição do peixe na família, isto é, o fato de diferentes membros da família consumirem diferentes qualidades de peixe, pode explicar parcialmente tais divergências. Da mesma forma, os fatores relacionados ao metabolismo do mercúrio orgânico, com decorrente acumulação no cabelo, devem ser também investigados. São conhecidos alguns mecanismos de proteção toxicológica para os metais que envolvem a ligação do metal a proteínas complexas - metalotioneínas. Tais complexos proteínametal são retidos nas células (gastrintestinais, hepáticas e renais), sem causar injúrias (Goyer, 1991).

As elevadas concentrações de mercúrio em crianças merecem maiores investigações, principalmente quando se considera que o mercúrio tem sido lançado na área desde o final da década de 70. É provável que, além das atuais exposições através do consumo de peixe, muitas das crianças menores de 15 anos de idade tenham sido também expostas ao mercúrio durante a vida intra-uterina e o aleitamento materno. Tais exposições não estão refletidas nas presentes análises, dado que os respectivos segmentos de cabelo já não estão mais disponíveis nestes indivíduos.

Como grupo controle, foram analisadas amostras de cabelo de 41 indivíduos residentes em Porto Velho, incluídos no grupo por referirem consumo esporádico (menos de 2 a 3 vezes por mês) de pescado local. Destes, $76 \%(n=31)$ apresentaram menos que 2,0 ppm de mercúrio total nas amostras de cabelo, enquanto o restante atingiu o pico de 4,5 ppm. Para contrastar, apenas $1 \%(n=4)$ dentre os ribeirinhos apresentaram concentração de mercúrio inferior a 2,0 ppm.

Os resultados aqui apresentados diferem de estudos anteriores realizados na Amazônia, uma vez que a população em estudo não está envolvida na atividade garimpeira, sendo a mesma composta por ribeirinhos que consomem grande quantidade de peixe. Dentre as 150 famílias entrevistadas, $79 \%$ referem consumo diário de peixe durante o verão local, enquanto $17 \%$ referem consumo de 3 a 5 dias por semana na mesma estação. Além disso, conforme mencionado, no presente estudo foi realizada a análise para mercúrio total e inorgânico. A maioria dos trabalhos realizados na Amazônia sobre exposição ao mercúrio apresenta amostras de cabelo provenientes de garimpeiros, isto é, indivíduos expostos ao mercúrio metálico na forma de vapor, cujas análises em amostras cabelo não incluem especiação de mercúrio. Por exemplo, Malm et al. (1990) encontraram concentrações de mercúrio total em nove amostras de cabelo provenientes de garimpeiros do Alto Madeira variando entre 0,2 e $40 \mathrm{ppm}$. Na mesma região - Alto Madeira —, Silva-Forsberg et al. (s/d) verificaram que, em $57 \%$ dos indivíduos investigados (garimpeiros, $n=54$; cozinheiras, $n=7$; ribeirinhos, $n=2$; e serviços diversos no garimpo, $n=2)$, a concentração de mercúrio total em amostras de cabelo foi maior que $6 \mathrm{ppm}$, atingindo o máximo de $111 \mathrm{ppm}$. Couto et al. (1988) analisaram 35 amostras de cabelos de garimpeiros de Cumaru, Cachoeiro e dos índios Kayapó, estado do Pará, e obtiveram concentrações médias, por localidade, de 5,2, 11,5 e 4,7 ppm de mercúrio total, respectivamente. Tais exposições são apresentadas por estes autores de acordo com a atividade ocupacional dos indivíduos amostrados no garimpo (comprador de ouro, gerente de dragas, proprietário), mas não de acordo com o consumo de peixe. Fernandes et al. (1990) apresentam concentrações de mercúrio em amostras de cabelo provenientes de 27 indivíduos inferiores a 4,8 ppm, além de dois indivíduos com concentrações de 11,2 e 15,7 ppm. Tais autores mencionam apenas que os indivíduos foram incluídos na amostra de acordo com características relativas a residência próximo à área de garimpo, consumo de pescado local e consumo de água fluvial sem tratamento prévio. Diferentemente, Oliveira et al. (1990) incluem 24 amostras de cabelo de ribeirinhos de Barão de Melgaço e 14 de Poconé, Mato Grosso, e acrescentam informações sobre o consumo de peixe e concentração de mercúrio em peixes. No primeiro grupo (Barão de Melgaço), a média de mercúrio total foi de 5,6 ppm, sendo a concentração máxima 22,6 ppm. Nesta localidade, $46 \%$ dos indivíduos amostrados para coleta de cabelo referiram consumir peixe mais que cinco vezes por semana. Em Poconé, a média foi de 0,8 e a variação, de 0,3 a 3,1 ppm sendo que apenas $21 \%$ dos indivíduos referiram 
consumir peixe mais do que cinco vezes por semana.

Outros trabalhos relativos à exposição ao mercúrio orgânico, através do consumo de sementes de trigo tratadas com fungicidas organomercuriais, no Iraque, e do consumo de peixe contaminado, em Minamata, Japão, revelam que $5 \%$ da população exposta pode manifestar sintomas clínicos iniciais de exposição ao mercúrio orgânico (parestesia) quando amostras de cabelo apresentam concentrações de 50 a 125 ppm de mercúrio (WHO, 1976). No presente estudo, oito (3\%) indivíduos apresentaram concentrações superiores a $50 \mathrm{ppm}$, conforme já mencionado.

Os níveis mínimos de exposição intra-uterina causadores de efeitos adversos em crianças são ainda pouco conclusivos (McKeown-Eyssen et al., 1983). Porém, estudos recentes têm demonstrado que a deficiência em testes psicológicos manifestada por crianças aos seis anos de idade pode estar associada à exposição ao mercúrio, com níveis de 13 a 15 ppm no cabelo materno, durante a gravidez (Kjellstrom et al., 1989).

Os resultados disponíveis, apesar de não serem conclusivos, são fortes indicadores de que a população em estudo encontra-se seriamente ameaçada. Conforme mencionado, sendo os fetos mais sensíveis a menores níveis de concentração de mercúrio orgânico, as crianças geradas sob as atuais condições constituem especial grupo de risco.

Medidas de intervenção se fazem urgentes, incluindo assistência médica aos casos de elevadas concentrações de mercúrio. $\mathrm{O}$ uso do mercúrio na atividade garimpeira deve ser controlado, de forma que se evite o seu despejo no ambiente. Campanhas de saúde pública podem providenciar orientação às populações quanto ao consumo de pescado, de acordo com os níveis de concentração de mercúrio nas diferentes espécies ictiológicas.

\section{AGRADECIMENTOS}

Os autores agradecem à comunidade ribeirinha pela colaboração, interesse e hospitalidade manifestados no decorrer do trabalho; aos entrevistadores Denilde Cespede Pereira, Maria
Irenir de Sousa e Ibaldeci dos Santos Ferreira, alunos dos cursos de Enfermagem e Geografia da Universidade Federal de Rondônia; e a Paulo R. M. Silva e Fatima A. B. Barreto, do Departamento de Química da Universidade de Brasília, pela colaboração nas análises de mercúrio realizadas nas amostras aqui apresentadas.

\section{RESUMO}

BOISCHIO, A. A. P. \& BARBOSA, A. Exposição ao Mercúrio Orgânico em Populações Ribeirinhas do Alto Madeira, Rondônia, 1991: Resultados Preliminares. Cad. Saúde Públ., Rio de Janeiro, 9 (2): 155160, abr/jun, 1993.

O presente trabalho discute os resultados da concentração de mercúrio total em amostras de cabelo da população ribeirinha do Alto Madeira, Rondônia. Dentre as 311 amostras de cabelo analisadas, $51 \%(\mathrm{n}=158)$ apresentam concentrações de mercúrio acima de $10 \mathrm{ppm}$. A variabilidade nas exposições ao mercúrio é ilustrada por uma família onde cinco indivíduos apresentam as concentracões de mercúrio mais elevadas (entre 90,6 e 303,1 ppm), enquanto em outros cinco indivíduos da mesma unidade familiar tais concentrações variam entre 7,0 e 13,3 ppm. Especial atenção deve ser dirigida à população feminina em idade reprodutiva, dado que o feto apresenta maior vulnerabilidade a menores exposições ao mercúrio orgânico. Neste estudo, 53\% $(n=37)$ da população feminina em idade reprodutiva (grupo etário 4) apresentam concentração de mercúrio superior a 10 ppm.

Palavras-Chave: Exposição Ambiental; Mercúrio; Cabelo; Análise, Pescado, Consumo

\section{REFERÊNCIAS BIBLIOGRÁFICAS}

COUTO, R. C. S. C.; CÂMARA, V. M. \& SABROZA, P. C., 1988. Intoxicação mercurial: resultados preliminares em duas áreas garimpeiras PA. Cadernos de Saúde Pública, 4: 301-315.

FAGERSTROM, T. \& JERNELOV, A., 1972. Some aspects of the quantitative ecology of mercury. Water Research, 6: 1193-1202. 
FERNANDES, R. S.; GUIMARÃES, A. F.; BIDONE, E. D.; LACERDA, L. D. \& PFEIFFER, W. C., 1990. Monitoramento do mercúrio na área do projeto Carajás. In: Seminário Nacional Riscos e Consequêencias do Uso do M ercúrio (S. Hacon; L. D. Lacerda; W. C. Pfeiffer \& D. Carvalho, eds.), pp. 211-228, Rio de Janeiro: Finep/UFRJ.

GOYER, R. A., 1991. Toxic effects of metals. In: Toxicology, The Basic Science of Poisons (M. Amdur; J. Doull \& C. Klaassen, eds.), pp. 623680, New York: Pergamon Press.

IPCS (International Programme on Chemical Safety), 1990. M ethylmercury-Environmental Health Criteria 101. Geneva: World Health Organization.

KJELLSTROM, T.; KENNEDY, P.; WALLIS, S.; STEWART, A.; FRIBERG, L.; LIND, B.; WUTHERSPOON, T. \& MANTELL, C., 1989. Physical and Mental Development of Children with Prenatal Exposure to Mercury from Fish Stage 2: Interviews and Psychological Tests at Age 6. Sweeden: National Swedish Environmental Protection. (Board Report 3642)

MAGOS, L. \& CLARKSON, T. W., 1972. Atomic absorpiton determination of total, inorganic and organic mercury in blood. Journal of the Association of Official A nalytical Chemists, 55: 966971.

MALM, O.; PFEIFFER, W. C.; SOUZA, C. M. M. \& RETHER, R., 1990. Mercury pollution due to gold mining in the Madeira river basin, Brazil. A mbio, 19: 11-15.
McKEOWN-EYSSEN, G. E. \& RUEDY, J., 1983. Prevalence of neurological abnormality in Cree Indian exposed to methylmercury in Northern Quebec. Clinical \& Investigative Medicine, 6: 161-169.

OLIVEIRA, E. F.; SILVA, E. C. \& OZAKI, S. K., 1990. Mercúrio via cadeia trófica na Baixada Cuiabana: ensaios preliminares. In: Seminário Nacional Riscos e Conseqüências do Uso do Mercúrio (S. Hacon; L. D. Lacerda; W. C. Pfeiffer \& D. Carvalho, eds.), pp. 202-210, Rio de Janeiro: Finep/UFRJ.

PFEIFFER, W. C.; MALM, O.; SOUZA, C. M. M.; LACERDA, L. D. \& SILVEIRA, E. G., 1990. A ameaça do mercúrio nos garimpos. Ciência Hoje, 11: 10-12.

SILVA-FORSBERG, M. C.; FORSBERG, B.; PIMENTEL, T. P. \& DINIZ, R., s/d. Contaminação mercurial humana no rio Madeira, Rondônia. Anais do II Congresso Latino-Americano de Ecologia. Caxambu, MG, Brasil. (No Prelo)

WHO (World Health Organization), 1976. Environmental Health Criteria 1: Mercury. Geneva: World Health Organization 